EUROPHYSICS LETTERS

Europhys. Lett., (), pp. ()

\title{
Phonon-Phason coupling in icosahedral quasicrystals
}

\author{
W.-J. Zhu(*) AND C. L. Henley \\ Dept. of Physics, Cornell University, Ithaca NY 14853-2501 \\ (received ; accepted ) \\ PACS. 61.44Br - Quasicrystals. \\ PACS. 62.20Dc - Elasticity, elastic constants. \\ PACS. 61.10Dp - Theories of X-ray diffraction and scattering. \\ PACS. 71.15Pd - Molecular dynamics calculations (Car-Parrinello) and other numerical simu- \\ lations.
}

\begin{abstract}
From relaxation simulations of decoration-based quasicrystal structure models using microscopically based interatomic pair potentials, we have calculated the (usually neglected) phonon-phason coupling constant. Its sign is opposite for the two alloys studied, $i$-AlMn and $i$ - $(\mathrm{Al}, \mathrm{Cu}) \mathrm{Li}$; a dimensionless measure of its magnitude relative to the phonon and phason elastic constants is of order $1 / 10$, suggesting its effects are small but detectable. We also give a criterion for when phonon-phason effects are noticeable in diffuse tails of Bragg peaks.
\end{abstract}

The elastic description of an icosahedral quasicrystal includes both "phonon" (ordinary) strain and "phason" strain. "Phason" strain parametrizes the way in which the local tile distribution deviates from icosahedral symmetry, as forced by the constraint of packing with surrounding tiles. Correspondingly there are two easily measured "phonon" elastic constants (ordinary bulk and shear modulus), and two "phason" elastic constants. The latter have been

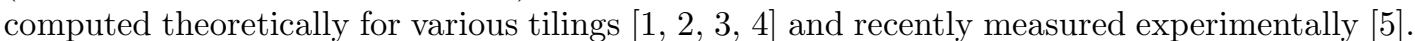

There remains a cross-term with one elastic constant, the "phonon-phason" coupling $\Gamma^{\|, \perp}$, which has not previously been measured experimentally or theoretically. The constant $\Gamma^{\|, \perp}$ enters the shape of the diffuse tails around Bragg peaks [6], can drive a quasicrystal phase unstable with respect to a related crystal phase [7, 8], and affects the strain fields of dislocations in quasicrystals, which have been studied by diffraction contrast in transmission electron microscopy [9, 10]. Previous authors [5, 6, 10, 11] simply assumed $\Gamma^{\|, \perp}$ was small compared with the other elastic constants.

The present work is the first attempt to compute $\Gamma^{\|, \perp}$ theoretically. As explained below, we extract it numerically from simulations in which we relax the atomic positions of a finite "periodic approximant" of the quasicrystal and measure its spontaneous shear distortion in response to the its intrinsic phason strain, for several different approximants. This was carried out for moderately realistic models of both $i$-AlMn and $i$-(Al, $\mathrm{Cu}) \mathrm{Li}$ (modeled as a

$\left({ }^{*}\right)$ Present address: IBM T.J. Watson Research Center P.O. Box 218, Yorktown Heights, NY 10598

Typeset using EURO-TEX 
pseudo-binary), representing the two major classes of quasicrystal. (Here "model" denotes a combination of a tiling, an atomic decoration rule, and a microscopically based pair potential.) In our conclusion, we crudely estimate ratios which determine the experimental observability of $\Gamma^{\|, \perp}$.

Elastic theory. - This is formulated in terms of two kinds of strain fields: the "phonon" (ordinary) strain tensor has components $u_{i j}=\left(\partial_{i} u_{j}+\partial_{j} u_{i}\right) / 2$ where $\mathbf{u}(\mathbf{r})$ is the phonon displacement. The phason strain components are $v_{i j}=\partial_{i} v_{j}$. Here $\mathbf{v}(\mathbf{r})$ is the phason (also called "perp") displacement field, defined for quasicrystals and/or tilings; it can be constructed for any configuration of the tiles [12, 13], and parameterizes the local imbalance in tiles of different orientations. Periodic approximant structures [15] of the quasicrystalline state necessarily acquire a non-zero phason strain.

Group theory [6, 14] permits the following terms in the elastic free energy (in the notation of [7], and adopting the summation convention):

$$
\begin{aligned}
F & =F_{\text {phonon }}+F_{\text {phason }}+F_{\text {phonon-phason }} \\
F_{\text {phonon }} & =\int d^{3} \mathbf{r} \frac{1}{2}\left[\lambda u_{i i}^{2}+\mu u_{i j} u_{i j}\right] \\
F_{\text {phason }} & =\int d^{3} \mathbf{r} \frac{1}{2}\left[K_{1} v_{i j} v_{i j}+K_{2}\left\{v_{k k}^{2}-\frac{4}{3} v_{i j} u_{i j}+\left[\left(\tau v_{12}+\tau^{-1} v_{21}\right)^{2}+\text { perms }\right]\right\}\right] \\
F_{\text {phonon-phason }} & =\int d^{3} \mathbf{r} \Gamma^{\|, \perp}\left\{\left[v_{11}\left(u_{11}-\tau u_{22}+\tau^{-1} u_{33}\right)+2 u_{23}\left(\tau^{-1} v_{23}-\tau v_{32}\right)\right]+\text { perms }\right\}
\end{aligned}
$$

Here "perms" represents two terms obtained from the preceding term (within the same parenthesis) by cyclic permutations of the indices (123). The term $F_{\text {phonon }}$ is the free energy of any isotropic harmonic solid with strain. It has two independent Lamé constants $\lambda$ and $\mu$, the latter being the shear modulus. Its "phason" analog is the $F_{\text {phason }}$ term with elastic constants $K_{1}$ and $K_{2}$. The $F_{\text {phonon-phason }}$ term couples the shear strain with one representation of the phason strain.

As in ordinary crystals, the elastic free energy describes the effects of thermal fluctuations on diffraction peaks, as well as possible soft-mode instabilities of the quasicrystalline state.

One application of elastic theory is to dislocations in a quasicrystal, since any Burgers vector necessarily has both phonon and phason components. The smaller the ratio $K_{1} / \mu$, the smaller the (expected) phonon component and the larger the phason component for a stable dislocation [9, 10]. This might be modified by a nonzero $\Gamma^{\|, \perp}$.

Method for measuring phonon-phason coupling $\Gamma^{\|, \perp}$.-

Given any structure model, $\Gamma^{\|, \perp}$ may be measured from the intrinsic distortion common to all approximant tilings. Its origin is the (known) nonzero uniform phason strain $\left(v_{i j}\right)$ characteristic of each periodic crystalline approximant. Given $\left(v_{i j}\right)$, the elastic theory then predicts a corresponding nonzero uniform (phonon) shear strain. Concretely, this means each tile spontaneously distorts a bit proportional to $\Gamma^{\|, \perp}$ times $\left(v_{i j}\right)$, so that tile edges in different directions are no longer quite related by icosahedral symmetry.

Consider in particular an orthorhombic (pseudo-tetragonal) approximant with $v_{11}=v_{22} \neq$ $v_{33}$, and all other $v_{i j}=0$. We allow it to distort with fixed-volume shears $u_{11}=u_{22}=$ $-\epsilon / 2, u_{33}=\epsilon$, so that the bulk modulus $\lambda$ does not contribute to the energy. Under this constraint, the minimum free energy occurs at

$$
\epsilon=\epsilon_{\min } \equiv-\frac{\Gamma^{\|, \perp}}{2 \mu}\left(v_{33}-v_{11}\right)
$$


Given a particular approximant, we can numerically compute the relaxed structural energy difference $E_{r e l}(\epsilon)$ for several choices of shear magnitude $\epsilon$. Obtaining $\epsilon_{\min }$ and $\mu$ by fitting

$$
E_{\mathrm{rel}}(\epsilon)=\frac{1}{2} \mu\left(\epsilon-\epsilon_{\text {min }}\right)^{2}+\text { const, }
$$

we finally deduce $\Gamma^{\|, \perp}$ using (2).

Specification of the models. - Even a crude estimate of the phonon-phason coupling requires sensible atomic models and realistic interatomic potentials [16, 17], otherwise the structure is not very stable and likely to rearrange irreversibly after small perturbations. There are two important and different icosahedral structure types: the Frank-Kasper class and the Al-transition metal class, represented by $i$ - $(\mathrm{Al}, \mathrm{Cu}) \mathrm{Li}$ and $i$-AlMn. We begin with the specification of the structural model, the first kind of input to our calculation.

The atomic positions in the unit cell to be relaxed are determined by three partly independent structural factors: tiling, decoration, and approximant. 16. The simplest icosahedral tiling geometry is the well-known three-dimensional rhombohedral tiling (3DRT) with edges along 5-fold symmetry axes [15, 18]. The Canonical Cell Tiling (CCT) [19] is an alternative tiling of 4 larger cells, which permits adjustments of the decoration to eliminate high-energy environments.

In the AlMn system we have used one a representative decoration rule from each of three families of decoration rules for the CCT 116, 17] These are labelled DF, DS, and LS in the scheme of [16], according to their tendency to dense (D) or loose (L) space-filling by the atoms, and their space-group symmetry ( $\mathrm{S}=$ Simple Icosahedral or $\mathrm{F}=$ Face-Centered Icosahedral). ${ }^{1}$ ) In the $(\mathrm{Al}, \mathrm{Cu}) \mathrm{Li}$ system. we only use the "Henley-Elser" (HE) decoration of the 3DRT 18] the version in which rhombohedra are grouped into disjoint rhombic dodecahedra wherever possible). The HE decoration may also be applied to the CCT (which can be decomposed into a special case of the 3DRT [19]).

Having chosen the tiling type, we may select among the available anisotropic approximants. The lattice parameters for the unit cell of quasicrystal approximants are constrained to have only lengths which are powers of $\tau$ times the quasi-lattice parameter [15]. We simplify the conventional notation " $p / q$ " 15] for a rational approximant of $\tau$ by only using " $p$ " in our labelling. Thus, the anisotropic approximants are, ordered by increasing system size, 112, 221, $223,331,332, \ldots$ [2, 20]. In the CCT case, some of these cannot be tiled by the 4 CCT cells; in particular, there is no approximant between 331 and 335 [3].

Phason strain will be measured in units where the the rhombohedron edge length $a_{R}$ is taken to be unity in real space, as well as the corresponding edge in "perpendicular" space [13]. Thus $v_{\alpha \alpha}=\tau^{-3},-\tau^{-4}, \tau^{-5}$ when the $\alpha$ entry in the approximant's label is 1,2 , and 3 respectively.

The second input in the specification of a model is the choice of pair potential. This is the only feasible approach to the energetics, since the system size of atomic structures in typical quasicrystal approximants is orders of magnitude larger than the maximum allowed for $a b$ initio calculations.

We adopted available well-tested potentials, constructed using linear response theory [21], for the binary AlMn 17] and the pseudo-binary $(\mathrm{Al}, \mathrm{Cu}) \mathrm{Li}$ 22 systems. $\left(^{2}\right)$ The AlMn pair

$\left({ }^{1}\right)$ We used variants DF1.1, DS1.1, and LS1.1; we have not tried the other variants possible within each family, which differ by changes of the chemistry or density on particular sites 16, 17]; a representative LF1.1 from the fourth family of decorations in [16] did not even show a quadratic $E_{\text {rel }}(\epsilon)$ function.

$\left({ }^{2}\right)$ Neither of these alloys is an equilibrium quasicrystal; the potentials for better quasicrystals were not yet available. 
TABLE I. - Computed optimal shear $\epsilon_{\text {min }}$, with shear modulus $\mu$ and phonon-phason coupling $\Gamma^{\|, \perp}$ in eV/atom. Text explains first three columns.

\begin{tabular}{|c|c|c|c|c|c|}
\hline Potential & Structure & Approximant & $\epsilon_{\min }$ & $\mu$ & $\Gamma^{\|, \perp}$ \\
\hline \multirow[t]{3}{*}{ AlMn } & $\mathrm{CCT}(\mathrm{DF})$ & 331 & $0.03 \%$ & 6.7 & 0.021 \\
\hline & CCT(DS) & 331 & $0.11 \%$ & 6.3 & 0.066 \\
\hline & $\mathrm{CCT}(\mathrm{LS})$ & 331 & $0.22 \%$ & 7.6 & 0.168 \\
\hline \multirow[t]{6}{*}{$(\mathrm{Al}, \mathrm{Cu}) \mathrm{Li}$} & $\mathrm{CCT}(\mathrm{HE})$ & $\begin{array}{lll}33 & 31\end{array}$ & $-1.00 \%$ & 1.9 & -0.187 \\
\hline & 3DRT(HE) & 112 & $1.13 \%$ & 2.1 & -0.146 \\
\hline & & 221 & $-0.81 \%$ & 2.2 & -0.110 \\
\hline & & 223 & $-0.16 \%$ & 2.4 & -0.063 \\
\hline & & 331 & $-0.80 \%$ & 2.2 & -0.177 \\
\hline & & 332 & $0.16 \%$ & 1.8 & -0.047 \\
\hline
\end{tabular}

potentials involve the conventional perturbation theory for the nearly-free electrons in the $\mathrm{Al}-\mathrm{Al}$ interaction, and Green's function treatment of $\mathrm{Mn} d$-bands [23]. For the $(\mathrm{Al}, \mathrm{Cu}) \mathrm{Li}$ system, ternary potentials were first constructed from pseudopotential-based perturbation theory and a tight-binding approximation of the $\mathrm{Cu} d$-orbitals, and then the pseudo-binary potentials were obtained through proper averaging to reflect the chemical disorder in the system [22]. We call such pair potentials "realistically oscillating" 17 because they feature Friedel oscillations out to several Angstroms. These potentials were tested in previous studies (see [17] and 22]); as a further check we applied the ( $\mathrm{Al}, \mathrm{Cu}) \mathrm{Li}$ potentials to various crystal structures related to the Frank-Kasper family, and reproduced a sensible phase diagram [24].

Calculations and results. - We first constructed the atomic structure for each combination of model and pseudo-tetragonal approximant shown in table I. (Its first three columns identify the alloy and potential, the tiling type (and decoration), and the approximant, using notations explained above. The next larger approximant would have be "335" which was too large for our relaxations.

For our selected pair potential, we always chose a cutoff slightly beyond $6 \AA$, so as to sufficiently include the first two potential wells [17]. We applied to the cell a uniform fixedvolume shear $\epsilon$ and then relaxed the positions (keeping the new cell shape fixed.) A modified conjugate gradient energy relaxation for the system converges to within $10^{-6} \mathrm{eV} /$ atom. We sampled a grid of $\epsilon$ values to derive the relaxed energy curve $E_{\text {rel }}(\epsilon)$ which was fitted to (3). Combining this with (2) we extracted the shear modulus $\mu$ and phonon-phason coupling $\Gamma^{\|,}$, expressed in table I in units of $\mathrm{eV} /$ atom.

For a given interatomic potential, the ordinary shear stiffness has reasonably consistent values. From table I, we first note $\mu \approx 7 \mathrm{eV} /$ atom in $i$-AlMn or $2 \mathrm{eV} /$ atom for $i$ - $\left.(\mathrm{Al}, \mathrm{Cu}) \mathrm{Li} . \mathrm{C}^{3}\right)$ The number density is about 6.5 atoms $/ a_{R}{ }^{3}$ in $i$-AlMn 16] or 7.8 atoms $/ a_{R}{ }^{3}$ in $i$ - $(\mathrm{Al}, \mathrm{Cu}) \mathrm{Li}[18]$, with $a_{R}$ about $4.6 \AA$ or $5.05 \AA$, respectively. This gives the predictions $\mu \approx 75 \mathrm{GPa}$ in $i$-AlMn or $20 \mathrm{GPa}$ in $i-(\mathrm{Al}, \mathrm{Cu}) \mathrm{Li}$, to be checked against the experimental values $\mu=65 \mathrm{GPa}$ in $i$-AlPdMn [25] or $41 \mathrm{GPa}$ in $i$-AlCuLi [26].

Less surely, the table shows $\left|\Gamma^{\|, \perp}\right| \sim 0.1 \mathrm{eV} /$ atom. Although the magnitude of the phononphason coupling is ill-determined in table I, its sign is quite clear and not universal: it is positive for AlMn and negative for $(\mathrm{Al}, \mathrm{Cu}) \mathrm{Li}$. We have no microscopic explanation for these signs, and so cannot tell whether they hold generally for Al-TM and Frank-Kasper type quasicrystals.

$\left(^{3}\right)$ Note that $i$-AlMn has a larger $\mu$ because its Al-Al potential has an energy scale about twice as big as in the $(\mathrm{Al}, \mathrm{Cu}) \mathrm{Li}$ case; in turn, this is due to the different electron densities. 
The fitted $\Gamma^{\|, \perp}$ for $i$-(Al,Cu)Li from different approximants varies by a factor $\sim 4$. We attribute this to finite-size effects due to the smallness of the tractable approximants: the strain relaxation might be especially sensitive to certain rare environments, occurring only one or two (or no) times in various approximants, producing big fluctuations in the inferred $\Gamma^{\|, \perp}$.

We also relaxed all the 3DRT approximants of the $(\mathrm{Al}, \mathrm{Cu}) \mathrm{Li}$ structure using Roth's singlewell Lennard-Jones (LJ) potential with non-additive radii [28], rescaled to roughly match the first well of the realistic potentials. When the simulated binary alloy freezes under these LJ potentials, it is known to form a disordered quasicrystal of the Frank-Kasper class [28]. We found that $\Gamma^{\|, \perp}$ is much smaller in this case; our LJ results are tabulated in [27].

Our calculation depended on the assumption that the optimal shear is a function only of the approximant's phason strain, and insensitive to tile rearrangements (which do not change phason strain). Unfortunately, the only such comparison in our data set is between the 3DRT and $\mathrm{CCT}$ tilings in the $(\mathrm{Al}, \mathrm{Cu}) \mathrm{Li} 331$ approximant. In that case (see Table 1), similar elastic constants were indeed fitted.

We also adopted a crude and implausible "frozen" tiling assumption, that the energetic and entropic contributions to the elastic free energy are independent and linearly additive. The value of $\Gamma^{\|, \perp}$ might be substantially renormalized (and be temperature-dependent) if we allowed atom relaxations and tile reshufflings simultaneously.

Discussion. - We may now assess whether the phonon-phason term is strong enough to be measured experimentally, and even perhaps to affect the quasicrystal's stability. Near a Bragg vector $\mathbf{G}^{\|}$, the diffuse intensity (following Ishii's notation $[8$ ) is proportional to

$$
\left(\mathbf{G}^{\perp}, \mathbf{B}(\mathbf{q})^{-1} \mathbf{G}^{\perp}\right)-2\left(\mathbf{G}^{\|}, \mathbf{A}(\mathbf{q})^{-1} \mathbf{C}(\mathbf{q}) \mathbf{B}(\mathbf{q})^{-1} \mathbf{G}^{\perp}\right)+\left(\mathbf{G}^{\|}, \mathbf{A}(\mathbf{q})^{-1} \mathbf{G}^{\|}\right)
$$

We wish to estimate the relative importance of these three terms, representing respectively contributions from ordinary "phonons", from "phason" fluctuations, and from the phononphason cross term. The $3 \times 3$ matrices $\mathbf{A}(\mathbf{q}), \mathbf{B}(\mathbf{q})$, and $\mathbf{C}(\mathbf{q})$ are derived from the elastic free energy (11) and their formulas are given in eqs. (2.3)-(2.5) of [8]. Each matrix is a sum of terms proportional to the corresponding elastic constants and homogeneous of second order in $|\mathbf{q}|$. Crudely speaking, the matrices scale as $\|\mathbf{A}(\mathbf{q})\| \sim \mu|\mathbf{q}|^{2},\|\mathbf{B}(\mathbf{q})\| \sim K_{1}|\mathbf{q}|^{2}$, and $\|\mathbf{C}(\mathbf{q})\| \sim \Gamma^{\|, \perp}|\mathbf{q}|^{2}$. (This scaling neglects their strong angular dependences on $\mathbf{q} /|\mathbf{q}| ;$ it also ignores the distinctions between the two phonon or the two phason elastic constants, since indeed $\lambda \approx 2 \mu$ in a typical solid, and $\left|K_{2}\right| / K_{1}=O(1)$ for all cases quoted below.)

We may conveniently express important criteria using ratios which all have the same dimensions as phason strain. First, the phason fluctuations - the first term in (4) - surely dominate that sum, as assumed in the analysis of [5], thus

$$
\left|\mathbf{G}^{\perp}\right| /\left|\mathbf{G}^{\|}\right|>\left|\Gamma^{\|, \perp}\right| / \mu \text {. }
$$

Second, for the phonon-phason coupling to be measurable, it ought to be the dominant correction, i.e. the second term in (1) is the next largest. Thus

$$
\left|\mathbf{G}^{\perp}\right| /\left|\mathbf{G}^{\|}\right|>K_{1} /\left|\Gamma^{\|, \perp}\right| \text {. }
$$

Adopting the simplest sort of random-tiling scenario [12], all of the phason elastic term is part of the tiling's configurational entropy. Simulations find $K_{1} / T=0.81 a_{R}^{-3}$ with $K_{2} / K_{2}=0.61$ for the 3DRT [1, 2]. For a model approximating the CCT [4], they find $K_{1} / T$ is (at most) $2.5 a_{R}^{-3}$ with $K_{2} / K_{1} \approx-0.75$. [4) Furthermore, phason fluctuations fall out of

\footnotetext{
$\left({ }^{4}\right)$ A less reliable calculation, but for the true CCT, found $K_{1} \approx a_{R}^{-3}$. [3]
} 
equilibrium not so far below the melting temperature $T_{m}$, so we will replace $T \rightarrow T_{m} \approx 10^{3} \mathrm{~K}$ in these estimates. Thus, using the figures mentioned earlier for $a_{R}$ and the number density, a crude estimate for either alloy is $K_{1} \approx 0.03 \mathrm{eV} /$ atom, in the units of table I. (The experimental value of $K_{1}$ in $i$-AlPdMn [29] seems to be a factor of 10 smaller.)

The above quoted estimates for $\mu$ (for $i$-AlMn), $\Gamma^{\|, \perp}$, and $K_{1}$, when inserted into the right-hand sides of (5) and (6), give respectively 0.015 and 0.3 . Thus a Bragg peak may be found $\left(^{5}\right)$ such that the $\Gamma^{\|, \perp}$ term is the first correction. This correction would cause differences in shape of the (otherwise similar) diffuse scattering contours of two Bragg peaks with $\mathbf{G}^{\|}$in the same direction, as observed recently [29].

There is an additional correction (see e.g. [8]) to every term of (4), scaling with the relative order

$$
r \equiv\left|\Gamma^{\|, \perp}\right|^{2} / K_{1} \mu
$$

(provided we assume $K_{2}=O\left(K_{1}\right)$ and $\lambda=O(\mu)$, as in the previous discussion). Evidently $r$ is the (dimensionless) ratio of the right hand sides of (5) and (6). Using the elastic constants suggested above for $i$-AlMn and $i$-(Al,Cu)Li gives $r \simeq 0.05$ and $r \simeq 0.2$, respectively, uncertain by an order of magnitude either way. This correction provides a second way that $\Gamma^{\|, \perp}$ affects the diffuse scattering. Thus too, a version of $r$ shows up in the criterion for nondivergence of the fluctuations (and diffuse scattering), Widom's eq. (7): $C_{\mp}\left(\Gamma^{\|, \perp}\right)^{2} /(\lambda+2 \mu)\left(K_{1} \mp 4 K_{2} / 3\right)<1$ where $C_{-}=4 / 9$ and $C_{+}=4$.

The same ratio $r$ also determines the importance of $\Gamma^{\|, \perp}$ in the onset of instability to a uniform phason strain, as discussed in [7] and [8]. Such phason-elastic instabilities might generate the approximant phases - e.g. rhombohedral $R(A l C u F e)$ - typically occurring for compositions close to the quasicrystal's [8]. The stability criterion is given in Widom's eq. (5) [7]: $\left.\left.3\right|^{\|, \perp}\right|^{2} /\left(K_{1}-4 K_{2} / 3\right) \mu<1$. If $K_{2} / K_{1} \approx 0.6$, as in the random 3DRT [1] or in $i$-AlCuFe [29], the criterion says $r<0.07$. If our estimate of $r$ is valid for real $i$-AlCuFe, then it is close to a phonon-phason driven instability.

The smallness of the phonon-phason constant has been rationalized by the notion that it measures the "frustration" of a material, defined as the mismatch of the ideal interatomic spacings and the well radii of the potentials [12]. In the unfrustrated case, different terms of the interactions are independently satisfied by the atoms, which occupy tiling-like special positions. This permits good quasicrystal order, since the tile-tile energy may be low for more than one way of packing tiles along a face. At the same time, the lack of unbalanced forces on the atoms means there is less tendency for tile shapes to distort depending on the tile packing. In this way the "binary tiling", a two-dimensional atomic toy model [30], has a quite small $\Gamma^{\|, \perp}$ [31].

However, our results appear to contradict the above argument: among the three AlMn decorations of the CCT 331 approximant, the "LS" decoration is best adapted to the structure [17], yet it shows the highest $\Gamma^{\|, \perp}$. (We suppose this is somehow due to the small voids common in the "LS" structure, which allow greater atomic rearrangement in response to phonon or phason shears.) Similarly, on the 3DRT tilings with the $i-(\mathrm{Al}, \mathrm{Cu}) \mathrm{Li}$ structure, the $i-(\mathrm{Al}, \mathrm{Cu}) \mathrm{Li}$ potentials in Table I give a much larger phonon-phason coupling than the poorer LJ model (computed in 27]).

In conclusion, we have numerically estimated the phonon-phason coupling $\Gamma^{\|, \perp}$ for representatives of both major classes of icosahedral quasicrystal. We found it had a non-universal sign, and (with very large uncertainties) it was large enough in magnitude to be observable

$\left({ }^{5}\right)$ Bragg peaks exist with arbitrarily small $\left|\mathbf{G}^{\|}\right| /\left|\mathbf{G}^{\perp}\right|$, but with exponentially small intensities 13. 
and perhaps even to dominate the mechanism of the quasicrystal's instability to a uniform phason strain. To improve the calculations, a stable quasicrystal should be modeled and the fluctuations in small samples (due to variations in tiling configurations and in decoration rules) must be better understood.

We thank M. Krajči for the use of his relaxation code, J. Zou, A. Carlsson and R. Phillips for the AlMn potentials, and M. Windisch and J. Hafner for the $(\mathrm{Al}, \mathrm{Cu}) \mathrm{Li}$ potentials. We are grateful, for discussion and comments, to M. de Boisssieu, V. Elser, M. Widom, and especially to M. Mihalkovič who originally developed our decoration/relaxation methods. This work was supported by U.S. Dept. of Energy grant DE-FG02-89ER-45405.

\section{REFERENCES}

[1] L.-H. Tang, Phys. Rev. Lett. 64, 2390 (1990).

[2] L. J. Shaw, V. Elser, and C. L. Henley, Phys. Rev. B 43, 3423 (1991).

[3] M. E. J. Newman and C. L. Henley, Phys. Rev. B 52, 6386 (1995).

[4] M. Mihalkovič and C. L. Henley, unpublished.

[5] M. de Boissieu et al., Phys. Rev. Lett. 75, 89 (1995).

[6] M. V. Jarić and D. R. Nelson, Phys. Rev. B 37, 4458 (1988).

[7] M. Widom, Phil. Mag. A 64, 297 (1991).

[8] Y. Ishii, Phys. Rev. B 45, 5228 (1992).

[9] M. Wollgarten, et al, Phil. Mag. A 76, 455 (1997).

[10] W. Yang et al, Phil. Mag. A 77, 1481 (1998).

[11] J. Bachteler and H.-R. Trebin, Eur. Phys. J. B 4, 299 (1998).

[12] C. L. Henley, Quasicrystals: the State of the Art, World Scientific, Singapore, 1991.

[13] V. Elser, Phys. Rev. B 32, 4892 (1985).

[14] T. C. Lubensky, p. 199 in Introduction to Quasicrystals, volume 1 of Aperiodicity and Order, ed. M. V. Jarić (Academic Press, San Diego, 1988).

[15] V. Elser and C. L. Henley, Phys. Rev. Lett. 55, 2883 (1985).

[16] M. Mihalkovič et al, Phys. Rev. B 53, 9002 (1996).

[17] M. Mihalkovič et al Phys. Rev. B 53, 9021 (1996).

[18] C. L. Henley and V. Elser, Philos. Mag. Lett. B 53, L59 (1986).

[19] C. L. Henley, Phys. Rev. B 43, 993 (1991).

[20] W. Ohashi, Ph. D. thesis, Harvard University, 1989.

[21] J. Hafner, The Structures of Binary Compounds, page 147, Elsevier Science Publishers, 1989.

[22] M. Windisch et al Phys. Rev. B 49, 8701 (1994).

[23] J. Zou and A. E. Carlsson, Phys. Rev. Lett. 70, 3748 (1993); Phys. Rev. B 47, 3345 (1993).

[24] W.-J. Zhu and C. L. Henley, unpublished.

[25] Y. Amazit et al, Europhys. Lett. 20, 703 (1992).

[26] P. S. Spoor et al, Phys. Rev. Lett 75, 3462 (1995).

[27] W.-J. Zhu, Ph. D. thesis, Cornell University, 1999.

[28] J. W. Roth, R. Schilling, and H.-R. Trebin, Phys. Rev. B 51, 15833 (1995); J. Roth and C. L. Henley, Philos. Mag. A 75, 861 (1997).

[29] A. Letoublon and M. de Boissieu, unpublished.

[30] M. Widom et al, Phys. Rev. Lett. 58, 706 (1987).

[31] K. J. Strandburg, Phys. Rev. B 40, 6071 (1989). 